\title{
Identification of Enterobacter sakazakii from closely related species: The use of Artificial Neural Networks in the analysis of biochemical and I 65 rDNA data

\author{
Carol Iversen $^{\dagger 1}$, Lee Lancashire ${ }^{\dagger 1,2}$, Michael Waddington ${ }^{3}$, Stephen Forsythe ${ }^{1}$ \\ and Graham Ball*1,2
} \begin{abstract}
8NS, UK and ${ }^{3}$ Accugenix, 223 Lake Drive, Newark, DE 19702, USA
Email: Carol Iversen - carol.iversen@rdls.nestle.com; Lee Lancashire - lee.lancashire@ntu.ac.uk; Michael Waddington - MikeWaddington@accugenix.com; Stephen Forsythe - stephen.forsythe@ntu.ac.uk;

Graham Ball* - graham.balls@ntu.ac.uk

* Corresponding author †Equal contributors
\end{abstract}

Address: ${ }^{1 T h e}$ Nottingham Trent University, School of Biomedical and Natural Sciences, Clifton Campus, Clifton Lane, Nottingham, NG11 8NS UK, ${ }^{2}$ Loreus Ltd., Erasmus Darwin Building, College of Science and Technology, Nottingham Trent University, Clifton Lane, Nottingham, NG11

Published: 13 March 2006

BMC Microbiology2006, 6:28 doi:10.1186/147/-2180-6-28

This article is available from: http://www.biomedcentral.com/I47I-2/80/6/28

(C) 2006lversen et al; licensee BioMed Central Ltd.

This is an Open Access article distributed under the terms of the Creative Commons Attribution License (http://creativecommons.org/licenses/by/2.0), which permits unrestricted use, distribution, and reproduction in any medium, provided the original work is properly cited.

\begin{abstract}
Background: Enterobacter sakazakii is an emergent pathogen associated with ingestion of infant formula and accurate identification is important in both industrial and clinical settings. Bacterial species can be difficult to accurately characterise from complex biochemical datasets and computer algorithms can potentially simplify the process.

Results: Artificial Neural Networks were applied to biochemical and I6S rDNA data derived from 282 strains of Enterobacteriaceae, including 189 E. sakazakii isolates, in order to identify key characteristics which could improve the identification of $E$. sakazakii. The models developed resulted in a predictive performance for blind (validation) data of $99.3 \%$ correct discrimination between E. sakazakii and closely related species for both phenotypic and genotypic data. Three main regions of the partial rDNA sequence were found to be key in discriminating the species. Comparison between E. sakazakii and other strains also constitutively positive for expression of the enzyme $\alpha$-glucosidase resulted in a predictive performance of $98.7 \%$ for $16 \mathrm{~S}$ rDNA sequence data and $100 \%$ for phenotypic data.

Conclusion: The computationally based methods developed here show a remarkable ability in reducing data dimensionality and complexity, in order to eliminate noise from the system in order to facilitate the speed and reliability of a potential strain identification system. Furthermore, the approaches described are also able to provide valuable information regarding the population structure and distribution of individual species thus providing the foundations for novel assays and diagnostic tests for rapid identification of pathogens.
\end{abstract}

\section{Background}

Enterobacter sakazakii is an emergent pathogen associated with ingestion of infant formula milk that can lead to neonatal meningitis, necrotising enterocolitis and sepsis [1- 
5]. The International Commission for Microbiological Specifications for Foods [6] has ranked E. sakazakii as 'Severe hazard for restricted populations, life threatening or substantial chronic sequelae or long duration'. Therefore as there is no accepted gold standard methodology, the correct definition and identification of E. sakazakii is important for powdered infant formula manufacturers, as well as regulators, clinicians and epidemiologists.

In 1980, Farmer and co-workers [7] defined the species and described fifteen biogroups according to biochemical profiles. A defining characteristic has been activity of the $\alpha$-glucosidase enzyme. Consequently selective, differential media incorporating chromogenic or fluorogenic $\alpha$ glucosides such as the indolyl substrate 5-bromo-4chloro-3-indolyl- $\alpha$, D-glucopyranoside have been developed $[8,9]$. It has been reported that $100 \%$ of E. sakazakii ( $\mathrm{n}=129$ ) were positive for $\alpha$-glucosidase in comparison to $0 \%$ of other Enterobacter species $(\mathrm{n}=97)$ [10]; however a small number of other Enterobacteriaceae test positive for this enzyme.

Recently 16S rDNA sequencing has revealed that commercial biochemical test kits identified more than one species as 'E. sakazakii' [11], and that there are at least four genetically and biochemically distinct subgroups of E. sakazakii. In this study we applied Artificial Neural Networks (ANNs) [12-14] to biochemical and 16S rDNA data in order to identify key phenotypic characteristics and nucleotide sequences which could improve the identification of E. sakazakii in respect to, a) other Enterobacteriaceae, and b) non-E. sakazakii $\alpha$-glucosidase positive Enterobacteriaceae.

ANNs are adaptive, non linear forms of Artificial Intelligence (AI) inspired by the way the human brain learns and processes information in order to solve specific problems, such as pattern recognition and classification problems. The multi-layer perceptron (MLP) ANN is a form of feed-forward ANN architecture which contains several layers, with each node in one layer being connected to every node in the next by a series of weighted links. When used with the back-propagation algorithm, this type of ANN learns in a fashion analogous to the way learning in the human brain is carried out, that is, by example. In humans, learning involves minor adjustments being made to the synaptic connections between neurons, in ANNs, learning is achieved by updating the weights that exist between the processing elements that constitute the network topology.

ANNs were applied to biochemical and 16S rDNA data derived from 282 strains of Enterobacteriaceae, including 189 E. sakazakii isolates, in order to identify key characteristics which could improve the identification of E. sakaza- kii. Results show that ANNs have the potential to identify key features from the data, both for biochemical tests and sequence data. These key features may then be used to form the basis of novel rapid identification systems, which have the ability to classify samples by strain and eliminate the risk of false positive and negative results.

\section{Results}

Food, clinical and environmental isolates of E. sakazakii were shown by $16 \mathrm{~S}$ rDNA analysis to form four clusters. A summary of the main cluster groups is shown in Figure 1. The clusters that were positive for constitutive X- $\alpha$-glucoside metabolism were the four Enterobacter sakazakii groups, Buttiauxella noakiae, and two clusters of Enterobacteriaceae (groups 5 and 6 in Figure 1) that could not be matched, either by genomic or biochemical profile, to any currently named species.

\section{Model development and classification analysis}

A MLP ANN was used together with the back-propagation algorithm. Inputs to the network represented biochemical test results or sequence ID; two hidden nodes were used in the hidden layer for mathematical feature detection and a single output node was used to represent species class, with a class assignment of 1 representing E. sakazakii strains, and 2 representing all other strains. Models were developed utilising a random sample cross validation approach where 100 random training/test/validation submodels were run and evaluated. This repeated random sampling guarantees that all samples are treated as blind data a number of times, to ensure model generality and to enable confidence intervals to be calculated for each sample. For each of the models a full analysis was conducted including sensitivity analysis to determine the importance ratio of each input. This process removed all of the inputs singularly from the model. The error of predictions was then measured for each of the inputs removed. The sensitivity ratio was then calculated based on the performance with and without the given input. The hypothesis here is that if a given input is important its removal will have strong negative effect on predictive performance. Therefore a sensitivity ratio greater than one indicates an input whose removal is detrimental for the model. Additionally, the analysis of predictive performance was performed to evaluate model accuracy, sensitivity and specificity, and assessment of the raw ANN predictions was conducted for the positioning of individuals within the population.

\section{Phenotypic data}

Using the phenotypic data, the models developed resulted in a predictive performance for blind (validation) data of $99.3 \%$ (sensitivity of $100 \%$ and specificity of $97.6 \%$ ) correct discrimination between E. sakazakii and closely related species. The population distribution was also examined by plotting the individual predictions from the 


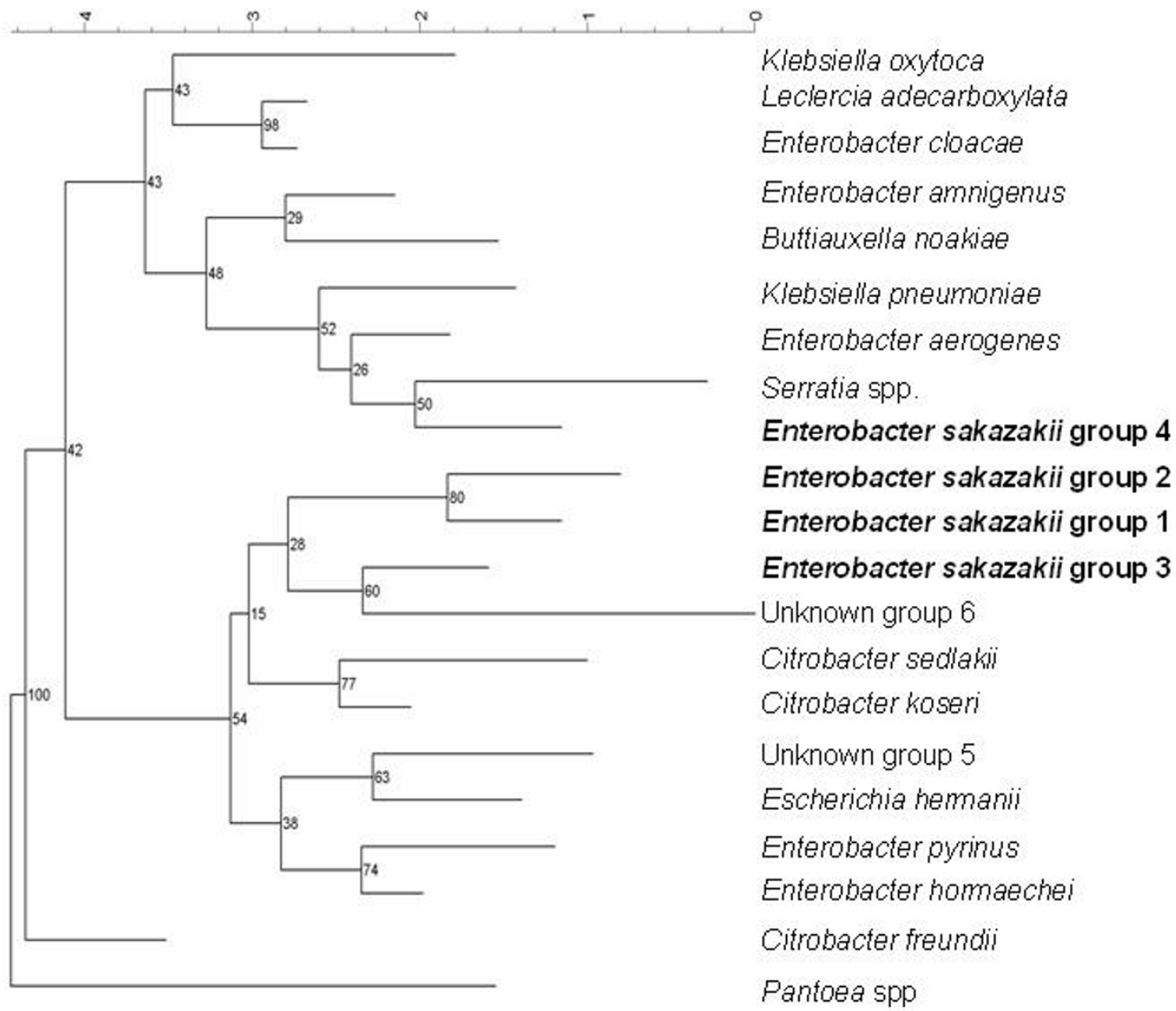

Figure I

Summary partial I6S rDNA sequence Neighbour Joining tree of E. sakazakii and related organisms. Bootstraps were derived from 1000 replicates and the Jukes-Cantor correction was applied.

ANN models (Figure 2). A model prediction of one indicates a sample is E. sakazakii whilst a two is indicative of another species, so as this value increases from one to two, the more characteristic a sample is of non-E. sakazakii origin. This distribution shows the variation that is present not only between the same strains, but also across species, which is why correct identification of pathogens can often be extremely difficult, with strains having the potential for frequent mutation and change.

\section{I6S rDNA sequence data}

The analysis was also repeated using $16 \mathrm{~S}$ rDNA sequence data to identify any areas of the sequence that could potentially be used to differentiate between the different species. The models developed produced predictive performances for blind (validation) data to an accuracy of once again, $99.3 \%$ (with sensitivity and specificity values of 99.5 and $98.9 \%$ respectively) of samples correctly identified as E. sakazakii or other species. There were three main regions of the sequence which were key in discriminating between the species. These regions all occur amongst regions that vary structurally among domains (see secondary structure Figure 3 ). Table 1 shows the 20 nucleotides with greatest relative importance and it is evident that they all appear to be derived from these focal positions in the sequence.

\section{Classification of $\alpha$-glucosidase positive strains}

Furthermore, the study was expanded in order to elucidate whether the ANNs could be used to differentiate between E. sakazakii and a number of other Enterobacteriaceae which test positive for constitutive metabolism of X- $\alpha$-glucoside. The same approach was used as above, with both phenotypic tests and 16S rDNA sequencing used as inputs 


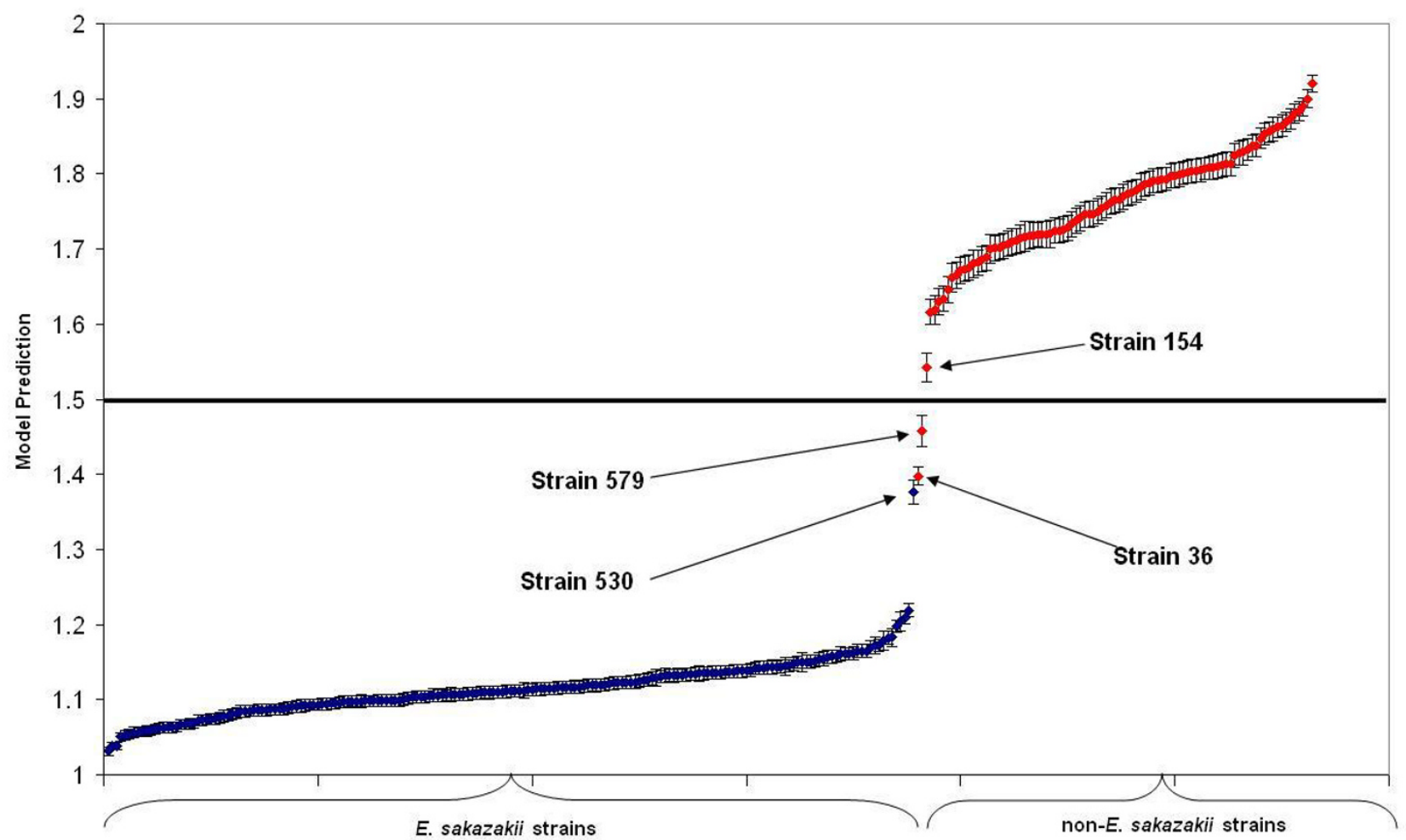

\section{Figure 2}

Population distribution of samples from the biochemical test data. Strains coloured blue represent E. sakazakii samples, whilst those in red represent non-E. sakazakii. The line at a predicted value of 1.5 represents the threshold for class prediction. Error bars indicate $95 \%$ confidence intervals, and labelled samples highlight those which were either misclassified or close to being so.

in the ANN models. Once again analysis using the ANN based approach proved to be extremely successful. Using the $16 \mathrm{~S}$ rDNA sequence data as inputs, the predictive performance of the ANN models was $98.7 \%$ (92.9 \% sensitivity, and $100 \%$ specificity). This improved further still when the biochemical test data results were used as inputs into the model. Here, $100 \%$ of the strains were correctly predicted into their respective classes, further highlighting the capabilities of ANN modelling in bacterial identification, which could potentially reduce the risk of false positive identification. The most relevant biochemical tests are summarised in Table 2, showing percent positive strains for $E$. sakazakii as well as other $\alpha$-glucosidase positive and negative Enterobacteriaceae.

\section{Discussion}

Models have been developed to identify (i) key biochemical tests and (ii) important areas of the DNA sequence which can be used in the accurate discrimination of $E$. sakazakii from other closely related species. Furthermore, the study was expanded to differentiate between $E$. sakazakii strains and other $\alpha$-glucosidase positive Enterobacte- riaceae. To date methods for the isolation and identification of $E$. sakazakii have used the $\alpha$-glucosidase reaction and production of yellow pigment as presumptive differentiating characteristics. However these methods can result in presumptive false positives due to groups of as yet undefined non-E. sakazakii Enterobacteriaceae which are also positive for both of these characteristics. Use of yellow pigment as a defining characteristic can also result in false negatives due to the occurrence of non-pigmented E. sakazakii and the occasional transient nature of this trait. While there is no single test that can be used to differentiate E. sakazakii from other species we identified biochemical profiles that may help to improve the likelihood of correct species identification.

Deriving a population distribution (Figure 2) from the analysis of the influence of biochemical tests in sample classification showed samples to appear in distinct clusters. This supports the interpretation of the partial $16 \mathrm{~S}$ rDNA clustering (Figure 1). The ANN model incorrectly identified two of the non-E. sakazakii strains as being $E$. sakazakii. The identities of these strains are highlighted in 


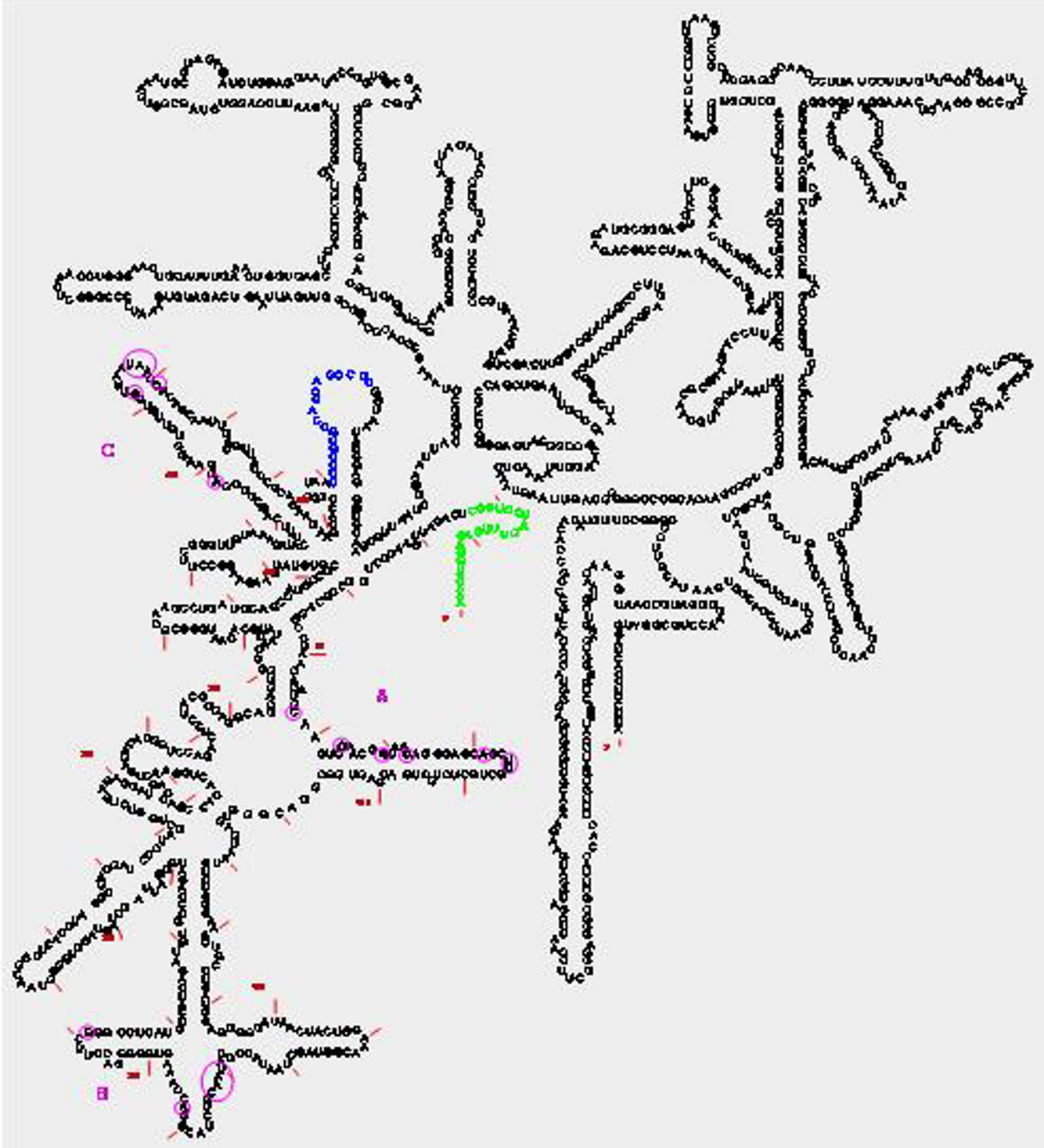

Figure 3

Secondary structure: small subunit ribosomal RNA of E. sakazakii NCTC I I 467. Nucleotides in green and blue represent primer sequence targets. Pink circles denote regions of importance as determined by Artificial Neural Networks.

Nucleotide numbering follows the Reference Numbering System used for E. coli J01695 [15]. Every 10th nucleotide is marked with a red tick mark and every 50th nucleotide is numbered. (Structure courtesy of Doug Smith, Accugenix, DE, USA). 
Table I: Top 20 nucleotides involved in classification from partial I6S rDNA data. Regions shown in this table are highlighted in Figure 3.

\begin{tabular}{lll}
\hline & $\begin{array}{c}\text { REGION } \\
\text { A }\end{array}$ & C \\
\hline 58 & 180 & 448 \\
64 & 181 & 462 \\
69 & 182 & 467 \\
72 & 183 & 468 \\
81 & 192 & 469 \\
84 & 211 & 471 \\
85 & & \\
\hline
\end{tabular}

Figure 2, and these samples may provide a basis for further studies because they are being incorrectly classified as a result of them displaying characteristics of both of the two groups, but are being determined to be more related to the E. sakazakii group. Alternatively, since the $16 \mathrm{~S}$ identification is based on differences between a number of nucleotide bases, the combinations of these is different for different species. The ANN models search for common elements of these bases, which are consistently represented in each class, and classifies based on these commonalities. Considering this, together with the incorrectly identified non- E. sakazakii strains, leads to the view that there may not be one base, or a series of bases, that are unique to E. sakazakii, and in some strains, such as those incorrectly identified by the model, common elements exist between $E$. sakazakii, and other strains.

Results from the analysis of the 16S rDNA data indicate that the key inputs identified were present in three distinct areas of the sequence and these areas were subsequently all regions that varied structurally among domains (Figure $3)$.

\section{Conclusion}

ANNs display their potential use in reducing model dimensionality and complexity, in order to facilitate the speed and reliability of a potential strain identification system. These methods are also able to provide valuable information regarding the population structure and distribution of individual species. These technologies may provide the foundations for novel assays and diagnostic tests for rapid identification of pathogens, and subsequently reducing the risk of incorrect diagnosis due to the occurrence of false positive and negative test results.

\section{Methods}

Genotypic and phenotypic data was collected for 282 strains of Enterobacteriaceae, including 189 E. sakazakii isolates and 39 other $\alpha$-glucosidase positive strains. Strains were from diverse food, clinical and environmental sources worldwide. Clinical isolates were from cases occurring over the last 25 years. At least one original strain from each of the biogroups described when the E. sakazakii species was designated were included [7].

\section{Phenotypic data}

Biochemical characteristics were derived from commercial test kits (API 20E and ID32E, bioMérieux UK Ltd.; Biolog GN2, Biolog, CA; and Microbact 24E, Oxoid UK Ltd.) and conventional manual tests as per standard protocols. Tests were performed in triplicate on separate days. Motility was determined at $37^{\circ} \mathrm{C}$ after $24 \mathrm{~h}$ and $48 \mathrm{~h}$ using motility medium (tryptose $10 \mathrm{~g} \mathrm{l}^{-1}, \mathrm{NaCl} 5 \mathrm{~g} \mathrm{l}^{-1}$, agar $5 \mathrm{~g} \mathrm{l}^{-1}, \mathrm{pH} 7.2$ \pm 0.2 . Acid production from carbohydrates was tested in phenol red broth base $\left(10 \mathrm{~g} \mathrm{l}^{-1}\right.$ peptone, $1 \mathrm{~g} \mathrm{l}^{-1}$ yeast extract, $5 \mathrm{~g} \mathrm{l}^{-1} \mathrm{NaCl}, 0.018 \mathrm{~g} \mathrm{l}^{-1}$ phenol red) with addition of filter-sterilized carbohydrate solution (final concentration $0.5 \%$ ). Gas production was determined by collection in Durham tubes. The methyl red test was performed at 48

Table 2: Biochemical tests for the differentiation of $E$. sakazakii.

\begin{tabular}{lccc}
\hline Biochemical Tests & E. sakazakii $(\mathrm{n}=189)$ & other $\alpha$-glucosidase positive strains $(\mathrm{n}=39)$ & other Enterobacteriaceae $(\mathrm{n}=54)$ \\
\hline$\alpha$-glucosidase & $100 *$ & 100 & 0 \\
Arginine dehydrogenase & 97 & 13 & 67 \\
Citrate & 99 & 15 & 80 \\
D-saccharic acid & 0 & 23 & 33 \\
Dulcitol & 8 & 80 & 28 \\
glucose-I-phosphate & 0 & 59 & 83 \\
glucose-6-phosphate & 0 & 46 & 82 \\
Lipase & 96 & 44 & 4 \\
Methyl Red & 5 & 95 & 57 \\
Ornithine decarboxylase & 91 & 0 & 74 \\
Pyruvate & 3 & 92 & 50 \\
Raffinose & 100 & 15 & 63 \\
Sucrose & 100 & 21 & 47 \\
Voges Proskauer & 96 & 0 & 44 \\
Yellow pigment & 98 & 90 & 28 \\
\hline
\end{tabular}

* denotes percent strains from the data set which were positive for the test 
$\mathrm{h}$ on cultures grown in MR-VP broth (VWR, 1.05712.0500). The Voges-Proskauer test was performed at $24 \mathrm{~h}$ by addition of $40 \%$ potassium hydroxide in water and 5\% 1-naphthol in ethanol to cultures grown in MR$\mathrm{VP}$ broth. Indole production was measured at $24 \mathrm{~h}$ by addition of Kovacs reagent ( $5 \mathrm{~g}$ p-dimethylaminobenzaldehyde, $25 \mathrm{ml} \mathrm{HCl}, 75 \mathrm{ml}$ pentanol-1-ol) or James Reagent (70542 bioMérieux) to cultures grown in Peptone Water (CM0009 Oxoid Ltd). Nitrate reduction was measured by addition of $1 \%$ sulphanilamide in $1 \mathrm{M} \mathrm{HCl}$ and $0.02 \% \mathrm{~N}-1$ naphthylene diamine $\mathrm{HCl}$ in water. Zinc dust was added to negative tubes to confirm the presence of unreduced nitrate. Constitutive metabolism of X- $\alpha$-glucoside was determined by formation of blue-green colonies on media containing 5-bromo-4-chloro-3-indolyl- $\alpha, \mathrm{D}$ glucopyranoside (Chromogenic Enterobacter sakazakii medium (DFI formulation) CM1055, Oxoid Ltd.; and ESIA, AES Laboratoire, France).

\section{Comparative I6S rDNA sequencing}

This was performed by Accugenix (Newark, DE, USA) using the MicroSeq ${ }^{\mathrm{TM}} 500$ 16S rDNA Bacterial Sequencing Kit (Applied Biosystems). DNA was prepared for PCR by quick-heat lysis by removing one colony into a tube of PrepMan Ultra ${ }^{\mathrm{TM}}$ (Applied Biosystems) and placed at $99^{\circ} \mathrm{C}$ for $10 \mathrm{~min}$. Two microlitres of genomic DNA was amplified in $50 \mu \mathrm{l}$ of a master mixture consisting of $0.4 \mu \mathrm{M}$ TGGAGAGTTTGATCCTGGCTCAG and TACCGCGGCTGCTGGCAC primers, $200 \mathrm{mM}$ deoxynucleoside triphosphates, PCR buffer, 0.3 U of AmpliTaq DNA polymerase, and $10 \%$ glycerol. PCR conditions were $95^{\circ} \mathrm{C}$ for $10 \mathrm{~min}$; 30 cycles each of $95^{\circ} \mathrm{C}$ for $30 \mathrm{~s}, 60^{\circ} \mathrm{C}$ for $30 \mathrm{~s}$, and $72^{\circ} \mathrm{C}$ for $45 \mathrm{~s}$; and a final step at $72^{\circ} \mathrm{C}$ for $10 \mathrm{~min}$. Purification of the PCR product to remove excess primers and nucleotides was performed using Montage $\mathrm{SEQ}_{96}$ filter plates (Millipore). Cycle sequencing was performed with the sequencing module, and after removal of excess dyes using Montage $\mathrm{SEQ}_{96}$ filter plates (Millipore), the labelled extension products were separated on an ABI 310016 capillary genetic analyzer (Applied Biosystems). Partial sequencing was performed for all isolates, the length of the partial rDNA was 528 nucleotides, and in addition the full sequence for the E. sakazakii type strain (NCTC 11467) was obtained.

The data was analysed using Bionumerics (Applied Maths, Belgium) to construct Neighbour Joining trees, bootstraps were derived from 1000 replicates and the Jukes-Cantor correction applied.

The full 16S sequence was used for the representation of the secondary structure of the small subunit ribosomal RNA of E. sakazakii NCTC 11467. Nucleotide numbering follows the Reference Numbering System used for E. coli J01695 [15].

\section{List of abbreviations}

AI Artificial Intelligence

ANN Artificial Neural Network

MLP Multi-Layer Perceptron

\section{Authors' contributions}

CI performed the biochemical characterizations, collated the test data and wrote the biochemical methods section and text relevant to E. sakazakii. LL co-developed and performed the computational analyses for the study, and drafted the manuscript. MW provided the $16 \mathrm{~S}$ sequencing and wrote the $16 \mathrm{~S}$ sequencing methods section. SF coordinated and managed the project. GB co-developed the analysis methods and co-ordinated the project. All authors read and approved the final manuscript.

\section{Acknowledgements}

The authors would like to thank the following for the provision of strains; Nestle Research Center, Lausanne, Switzerland; Health Products and Food Branch, Health Canada; CDC, Atlanta, USA; Children's Hospital Los Angeles CA, USA; Northern Foods, UK; Oxoid Ltd., Basingstoke, UK; Hospital Cěské Budéjovice, Czech Republic; Justus-Liebig-Universität Gießen, Germany; NCHT, Nottingham, UK; St. Radboud Nymegen, Netherlands. Cultures from national collections were either from the NCTC, London, UK; the NCIMB Ltd, Aberdeen, Scotland; or the ATCC, Manassas, VA 20108 USA

\section{References}

I. Muytjens HL, Zanen HC, Sonderkamp HJ, Kollee LA, Wachsmuth IK, Farmer J]: Analysis of eight cases of neonatal meningitis and sepsis due to Enterobacter sakazakii . J Clin Microbiol 1983, I 8: I |5-120.

2. Lai KK: Enterobacter sakazakii infections among neonates, infants, children, and adults: Case reports and a review of the literature. Medicine (Baltimore) 2001, 80: I I3-122.

3. van Acker J, de Smet F, Muyldermans G, Bougatef A, Naessens A, Lauwers $S$ : Outbreak of necrotizing enterocolitis associated with Enterobacter sakazakii in powdered milk formula. J Clin Microbiol 200I, 39:293-297.

4. Himelright I, Harris E, Lorch V, Anderson M: Enterobacter sakazakii infections associated with the use of powdered infant formula - Tennessee, 200 I. JAMA 2002, 287:2204-2205.

5. Iversen C, Forsythe S: Risk profile of Enterobacter sakazakii, an emergent pathogen associated with infant milk formula. Trends Food Sci Technol 2003, I4:443-454.

6. ICMSF: International Commission on Microbiological Specifications for Foods. Micro-organisms in Foods Number 7. Microbiological Testing in Food Safety Management. Kluwer Academic/Plenum Publishers; 2002.

7. Farmer JJ, Asbury MA, Hickman FW, Brenner DJ, The Enterobacteriaceae Study Group: Enterobacter sakazakii, new species of Enterobacteriaceae isolated from clinical specimens. Int J Syst Bacteriol 1980, 30:569-584.

8. Iversen $C$, Druggan $P$, Forsythe $S$ : A selective differential medium for Enterobacter sakazakii, a preliminary study. Int J Food Microbiol 2004, 96(2): I33-139.

9. Leuschner RG, Bew J: A medium for the presumptive detection of Enterobacter sakazakii in infant formula: Interlaboratory study. J AOAC Int 2004, 87(3):604-6I3.

10. Muytjens H, van der Ros-van de Repe J, van Druten HAM: Enzymatic profiles of Enterobacter sakazakii and related species with special reference to the alpha glucosidase reaction and reproducibility of the test system. J Clin Microbiol 1984, 20:684-686. 
II. Iversen C, Waddington M, On S, Forsythe S: Identification and phylogeny of Enterobacter sakazakii relative to Enterobacter and Citrobacter species. J Clin Microbiol 2004, 42(I I):5368-5370.

12. Ball G, Mian S, Holding F, Allibone RO, Lowe J, Ali S, Li G, McCardle $S$, Ellis IO, Creaser C, Rees RC: An integrated approach utilizing artificial neural networks and SELDI mass spectrometry for the classification of human tumours and rapid identification of potential biomarkers. Bioinformatics 2002, 18:395-404.

13. Lancashire LJ, Mian S, Ellis IO, Rees RC, Ball GR: Current developments in the analysis of proteomic data: Artificial neural network data mining techniques for the identification of proteomic biomarkers related to breast cancer. Curr Proteomics 2005, 2: 15-29.

14. Lancashire L, Schmid O, Shah H, Ball G: Classification of bacterial species from proteomic data using combinatorial approaches incorporating artificial neural networks, cluster analysis and principal components analysis. Bioinformatics 2005, 21:2191-2199.

15. Gutell Lab Comparative RNA Web Site [http://
[e www.rna.icmb.utexas.edu]

Publish with Biomed Central and every scientist can read your work free of charge

"BioMed Central will be the most significant development for disseminating the results of biomedical research in our lifetime. "

Sir Paul Nurse, Cancer Research UK

Your research papers will be:

- available free of charge to the entire biomedical community

- peer reviewed and published immediately upon acceptance

- cited in PubMed and archived on PubMed Central

- yours - you keep the copyright 